\title{
A Study on Ayurveda Poly Herbal Compound of Yogaratnakar (17 - A.D.) w.s.r. Rakta-Pradara (Abnormal Uterine Bleeding)
}

\author{
Research Article
}

\begin{abstract}
Nomina V Adwani ${ }^{1 *}$, Paramkush Rao $\mathbf{M}^{2}$
1. P.G. Scholar final Year, 2. Prof and HOD,

P.G. Dept of Dravyaguna, S.V. Ayurvedic College, Tirupati (A.P.)

Abstract

Rakta Pradara (Abnormal uterine bleeding) is commonest problem encountered by gynecologist in today's practice and incidence becomes higher with degree of civilization. Various treatments prescribed in modern medicine like HRT, Antifibrinolytic agents etc have not proved their definite efficacy Inspite of high price and side effect. The present study titled "Clinical study of Shatavari Yashti yoga on Rakta-Pradara w.s.r. to Yogaratnakar" was aimed to observe the efficacy of the trial drug Shatavari Yashti Yoga (Poly herbal compound) in Rakta Pradara (Abnormal uterine bleeding). The clinical study was conducted on 30 patients selected randomly and divided in to three groups based on the drug for the clinical trial. Group 1- Shatavari Yashti Yoga with Anupana Tandulodaka; Group 2 - Shatavari Yashti Yoga without Anupana and Group 3 - Placebo with Tandulodaka. The Clinical assessment was done on the basis of grading criteria with specific Symptomology of Rakta Pradara (Abnormal uterine bleeding) like Amount and duration of bleeding, intermenstrual bleeding, Pain, burning sensation, pallor, and weakness then mean scores levels of these Symptoms before and after the treatment of all three groups were subjected for student paired ' $t$ ' test for statistical analysis. Conclusion: The results were statistically and clinically significant not only to cure Rakta Pradara but also in improving general health of women.
\end{abstract}

Keyword: Rakta Pradara, Yogaratnakar, Shatavari Yashti Yoga, Tandulodaka.

\section{Introduction}

Raktapradara is an excessive or prolonged flow of blood occurring in menstrual or inter menstrual period as per manuscripts of Ayurveda it shows close resemblance with Abnormal uterine bleeding where following pattern of abnormal and excessive uterine bleeding is found like Menorrhagia - Prolonged or excessive bleeding occurs at regular intervals; Metrorrhagia - Irregular, frequent uterine bleeding of varying amounts but not excessive; Meno-Metrorrhagia - Prolonged or excessive bleeding at irregular intervals; Dysfunctional uterine bleeding (DUB) - Abnormal uterine bleeding not caused by pelvic pathology, medications, systemic disease or pregnancy, is the most common cause of abnormal uterine bleeding but remains a diagnosis of exclusion.(1)

All the above pathologies can be correlated with Rakta Pradara. So many

*Corresponding Author:

Nomina V Adwani,

PG scholar,

PG Department of PG Dravyaguna,

SV Ayurvedic College,

Tirupati - 517507

E,mail - dr.nomina.727@ gmail.com

Mob: +918801082202 preparations have been mentioned in ayurvedic classic for the treatment of Rakta Pradara. All these medications have certain fundamental Principles. These are Rakta shodhaka (blood purifier), Sthambhaka (Haemostatic), balya (reduces weakness) etc. Samprapti vighatana and effective control and cure in Rakta Pradara are the main with 
which the drugs were selected. In pathogenesis of Rakta Pradara Rasa, Rakta, and vata dosha are main responsible factors. Shatavari Yashti Yoga (Poly herbal compound) Plays an important role to alleviate this pathology, and effectively controls the bleeding. It also helps to cure the complications which occur due to heavy uterine bleeding (pallor, burning sensation, weakness). Shatavari Yashti Yoga is pure herbal compound, non hormonal, cost effective and harmless remedy.

Due to limitation of medical therapy as well as surgical therapy of modern science, it becomes necessity of the time to find out an effective harmless therapy with the help of Ayurveda to manage the conditions and keeping this in view the polyherbal compound Shatavari Yashti Yoga with Tandulodaka (SYT) from Yogaratnakar (classical lexicon of Ayurveda) from Pradara Chikitsa (chapter deals with gynecological problems)(2) is selected to conduct the study on most prevalent disorder in present times called Raktapradara(Abnormal Uterine bleeding).

\section{Aims and objectives}

1. To provide a simple, safe and non-hormonal herbal drug for the patients of Raktapradara (Abnormal uterine bleeding).

2. Evaluation of therapeutic efficacy of polyherbal compound Shatavari-Yashti Yoga.

3. To analyze the influence of vehicle (Anupana) i.e. Tandulodaka (Rice wash water) in the patients of Raktapradara (Abnormal uterine bleeding).

\section{Material and method}

The Material taken for the study were,

A) Drugs - Shatavari wet root (roots of Asparagus racemosus Willd.)

Yashtimadhu dry root (roots of Glycyrrhiza glabra Linn.)

Sita / Mishri (Sugar candy)

Vehicle (Anupana) - Tandulodaka (Rice wash water)

\section{Preparation of Shatavari Yashti Yoga}

Shatavari Swarasa (juice of roots of Asparagus racemosus Willd.) + Yashtimadhu churna (powder of roots of Glycyrrhiza glabra Linn) + Sita/mishri (Sugar Candy). Mix well all the contents then this Mixture was dried under hot air oven at $40{ }^{\circledR c}$ for 1 day. Next day it was grinded in hammer crusher and granulated for the convenience of drug administration purpose.

\section{Method - Study Design}

For the Present clinical Study total number of 39 patients were registered and screened in to 3 groups for the clinical trial. The effects of the therapies were screened in 30 patients who have completing the therapy. The patients were sourced from two different sources-

1) OPD \& IPD of PG Department of Dravya Guna from S.V. Ayurvedic Hospital, Tirupati.

2) By organizing weekly free camp in S. P.W. Junior Girls Hostel, Tirupati.

\section{Criteria for inclusion of the patients}

1. Age between Menarche and Menopause (12-50 yrs.)

2. Excessive bleeding during menstruation (change of more than 3 soiled pads/day)

3. Passing of large clots.

4. Prolonged Menstrual bleeding (more than 7 days)

5. Excessive bleeding for more than 2 consecutive cycles 
6. Intermenstrual Bleeding

\section{Criteria for exclusion of the patients}

1. Intrauterine growth such as Myomas, endometrial polyp etc.

2. Cancer of cervix and or uterus.

3. Hb less than $6 \mathrm{gm} \%$.

4. Any other systemic disorders likely to influence menstrual cycle.

5. Case undergoing treatment for any other serious illness.

6. Woman .using an IUCD/ OCP.

7. Venereal disease.

8. Systemic cause mentioned below will also be kept under exclusion criteria.

- Severe hypertension

- Liver dysfunction

- Congestive cardiac failure.

- Patient having bulky uterus

- Patients having bleeding from sites other than uterus

- Patients having coagulation disorder

\section{Investigations}

\begin{tabular}{|l|l|}
\hline Blood & $\mathrm{Hb} \%$, TLC, DLC ,ESR ,CT , BT. \\
\hline USG & To rule out uterine pathologies. \\
\hline
\end{tabular}

\section{CLINICAL ASSESSMENT}

\section{Subjective Assessment}

The patients undergone treatment were assessed for efficacy of Shatavari-Yashti Yoga on basis of grading criteria depicted below for improvement in specific symptomatology of Rakta Pradara(Abnormal uterine bleeding).

1. Bleeding amount in no. of pads

\begin{tabular}{|l|l|l|}
\hline Sr. no. & \multicolumn{1}{|c|}{$\begin{array}{l}\text { Bleeding Amount } \\
\text { No. of pads/ day }\end{array}$} & Grade \\
\hline 1. & $1-2$ pads/day & 0 \\
\hline 2. & $3-4$ pads/day & 1 \\
\hline 3. & $5-6$ pads/day & 2 \\
\hline 4. & 7and above pads/day & 3 \\
\hline
\end{tabular}

2. Duration of flow or menstrual period (In no of days)

\begin{tabular}{|l|l|l|}
\hline Sr. no. & \multicolumn{1}{|c|}{ Duration of Bleeding in no of days } & Grade \\
\hline 1. & $3-5$ days & 0 \\
\hline 2. & $6-7$ days & 1 \\
\hline 3. & $8-9$ days & 2 \\
\hline 4. & 9 and above days & 3 \\
\hline
\end{tabular}

3. Interval of Menstruation (in no. of days)

\begin{tabular}{|l|l|l|}
\hline Sr. no. & \multicolumn{1}{|c|}{$\begin{array}{c}\text { Interval of Menstruation } \\
\text { (Gap between two cycles) }\end{array}$} & Grade \\
\hline 1. & $25-30$ days & 0 \\
\hline 2. & $20-24$ days & 1 \\
\hline
\end{tabular}




\begin{tabular}{|l|l|l|}
\hline 3. & $15-19$ days & 2 \\
\hline 4. & Less than 15days & 3 \\
\hline
\end{tabular}

4. Pallor (Pandutava) (Hb \%)

\begin{tabular}{|l|l|l|}
\hline Sr. no. & \multicolumn{1}{|c|}{ Pallor } & Grade \\
\hline 1. & Normal $(>11 \mathrm{gm} \%)$ & 0 \\
\hline 2. & Mild $(9-11 \mathrm{gm} \%)$ & 1 \\
\hline 3. & Moderate $(7-9 \mathrm{gm} \%)$ & 2 \\
\hline 4. & Severe $(<7 \mathrm{gm} \%)$ & 3 \\
\hline
\end{tabular}

\section{Pain (Pain in abdomen / backache)}

\begin{tabular}{|l|l|l|}
\hline Sr. no. & Body ache & Grade \\
\hline 1. & No pain & 0 \\
\hline 2. & Mild pain & 1 \\
\hline 3 & Moderate pain & 2 \\
\hline 4. & Severe pain & 3 \\
\hline
\end{tabular}

\section{Burning Sensation (Daha)}

\begin{tabular}{|l|l|l|}
\hline Sr. no. & Burning sensation & Grade \\
\hline 1. & No burning & 0 \\
\hline 2. & Occasional burning & 1 \\
\hline 3. & Often mild burning & 2 \\
\hline 4. & Severe burning & 3 \\
\hline
\end{tabular}

\section{Weakness (Daurabalya)}

\begin{tabular}{|l|l|l|}
\hline Sr. no. & Weakness & Grade \\
\hline 1. & Feeling weakness Occasionally on doing Heavy work & 0 \\
\hline 2. & Feeling weakness After doing extra work then routine work & 1 \\
\hline 3 & Feeling weakness After doing Routine work & 2 \\
\hline 4. & Feeling weakness Even without Routine work & 3 \\
\hline
\end{tabular}

\section{TRIAL GROUPS}

In the present clinical Study total number of 39 patients were registered and 9 patients could not be followed So 30 patients who have completing the therapy are alienated in to 3 groups based on the drug for the clinical trial.

Group 1- SYT (Shatavari Yashti with Tandulodaka)

Group 2- SY (Shatavari Yashti Yoga)

Group 3-PL (Placebo with Tandulodaka)

\begin{tabular}{|l|l|l|l|l|l|}
\hline Sr. No & Group name & Dose & Vehicle & $\begin{array}{l}\text { Time of } \\
\text { administration }\end{array}$ & Duration \\
\hline 1.(SYT) & $\begin{array}{l}\text { Shatavari } \\
\text { Yashti Yoga }\end{array}$ & $5 \mathrm{gm}$ & Tandulodaka & $\begin{array}{l}\text { Twice a day before } \\
\text { meals }\end{array}$ & $\begin{array}{l}2 \text { menstrual cycle or } \\
2 \text { months. }\end{array}$ \\
\hline 2.(SY) & $\begin{array}{l}\text { Shatavari } \\
\text { Yashti Yoga }\end{array}$ & $5 \mathrm{gm}$ & - & $\begin{array}{l}\text { Twice a day before } \\
\text { meals }\end{array}$ & $\begin{array}{l}2 \text { menstrual cycle or } \\
2 \text { months. }\end{array}$ \\
\hline
\end{tabular}




\begin{tabular}{|l|l|l|l|l|l|}
\hline 3.(PL) & Tandulodaka & $50 \mathrm{ml}$ & - & $\begin{array}{l}\text { Twice a day before } \\
\text { meals }\end{array}$ & $\begin{array}{l}2 \text { menstrual cycle or } \\
2 \text { months. }\end{array}$ \\
\hline
\end{tabular}

Dose design

\begin{tabular}{|l|l|l|l|l|}
\hline Drug & Dose & Vehicle & $\begin{array}{l}\text { Time of } \\
\text { administration }\end{array}$ & $\begin{array}{l}\text { Duration of } \\
\text { treatment }\end{array}$ \\
\hline $\begin{array}{l}\text { Shatavari } \\
\text { Yashti Yoga }\end{array}$ & $5-\mathrm{gm}$ & Tandulodaka & $\begin{array}{l}\text { Twice a Day before } \\
\text { meals }\end{array}$ & $\begin{array}{l}2 \text { month with } \\
\text { follow up for } \\
\text { Next cycles }\end{array}$ \\
\hline
\end{tabular}

\section{Follow Up Study}

During the maneuver all patients were regularly analyzed and advised to attend O.P.D. after every 15 days till the end of trial and the patients from the Camp is monitored weekly. The notable changes were duly recorded. Laboratory investigations were carried out before and after the commencement of trial for purpose of inclusion criteria.

\section{Results}

The results were assessed before and after the treatment according to the positive changes in the subjective symptoms. Further statistical analysis was done by applying Student Paired ' $\mathrm{t}$ ' test and ' $\mathrm{P}$ ' value was calculated by referring to Fischer's table at the corresponding level of degree of freedom.(3) Total relief of the therapy was assessed on basis of percentage of relief obtained:

\begin{tabular}{|l|l|}
\hline \multicolumn{1}{|c|}{ Percentage of relief } & \multicolumn{1}{c|}{ Effect } \\
\hline More than $75 \%$ & Cured \\
\hline $51-75 \%$ & Markedly improved \\
\hline $26-50 \%$ & Moderately Improved \\
\hline Less than $25 \%$ & No improvement \\
\hline
\end{tabular}

In the present study 39, patients were registered out of which 9 patients dropped out during the trial and in remaining 30 patients the clinical study was completed. The study shows a marked difference in the subduing of symptoms in all the trial groups. The main symptoms studied were the amount and duration of bleeding, Pain, increased Hemoglobin levels, burning sensation, and generalized weakness. Thus it is clear from the study that the Shatavari Yashti Yoga helps in the Management of Rakta Pradara (Abnormal uterine bleeding).

\section{EFFECT OF THE TRIAL DRUG OF GROUP -1 (SYT) ON VARIOUS SUBJECTIVE SYMPTOMS}

Table no. 1 Showing Statistical analysis of amount of bleeding (in no. of pads)

\begin{tabular}{|c|c|c|c|c|c|c|c|c|}
\hline \multirow{2}{*}{ Symptoms } & \multicolumn{2}{|c|}{ Mean } & \multirow[b]{2}{*}{ Diff. } & \multirow[b]{2}{*}{ SD } & \multirow{2}{*}{ SE } & \multirow{2}{*}{$' t^{\prime}$} & \multirow[b]{2}{*}{$\mathbf{P}$} & \multirow[b]{2}{*}{ Results } \\
\hline & BT & AT & & & & & & \\
\hline $\begin{array}{l}\text { Amount of } \\
\text { Bleeding (in } \\
\text { no. of pads) }\end{array}$ & 3.9 & 0.2 & 3.7 & 0.8233 & 0.2603 & 14.212 & $\mathrm{P}<0.0001$ & $\begin{array}{l}\text { Extremely } \\
\text { significant }\end{array}$ \\
\hline
\end{tabular}


Table no. 2: Showing Statistical analysis of Duration of Bleeding (In no. of day)

\begin{tabular}{|c|c|c|c|c|c|c|c|c|}
\hline \multirow{2}{*}{ Symptoms } & \multicolumn{2}{|c|}{ Mean } & \multirow{2}{*}{ Diff. } & \multirow{2}{*}{ SD } & \multirow{2}{*}{ SE } & \multirow{2}{*}{$' t^{\prime}$} & \multirow{2}{*}{$\mathbf{P}$} & \multirow{2}{*}{ Results } \\
\hline & BT & $\mathbf{A T}$ & & & & & & \\
\hline $\begin{array}{l}\text { Duration of } \\
\text { Bleeding (in } \\
\text { no. of days) }\end{array}$ & 6.6 & 2.5 & 4.1 & 2.205 & 0.6403 & 6.403 & 0.0001 & $\begin{array}{l}\text { Extremely } \\
\text { significant }\end{array}$ \\
\hline
\end{tabular}

Table no. 3: Showing Statistical analysis of Interval of menstrual Bleeding (In no. of day)

\begin{tabular}{|c|c|c|c|c|c|c|c|c|}
\hline \multirow{2}{*}{ Symptoms } & \multicolumn{2}{|c|}{ Mean } & \multirow{2}{*}{ Diff. } & \multirow{2}{*}{ SD } & \multirow{2}{*}{ SE } & \multirow{2}{*}{ 't' } & \multirow{2}{*}{$\mathbf{P}$} & \multirow{2}{*}{ Results } \\
\hline & BT & AT & & & & & & \\
\hline $\begin{array}{l}\text { Intermenstrual } \\
\text { Bleeding (in no } \\
\text { of days) }\end{array}$ & 11.2 & 13.8 & -2.60 & 2.989 & 0.9452 & 2.751 & 0.0244 & Significant \\
\hline
\end{tabular}

Table no. 4: Showing Statistical analysis of Pain

\begin{tabular}{|c|c|c|c|c|c|c|c|c|}
\hline \multirow{2}{*}{ Symptoms } & \multicolumn{2}{|c|}{ Mean } & \multirow{2}{*}{ Diff. } & \multirow{2}{*}{ SD } & \multirow{2}{*}{ SE } & \multirow{2}{*}{ 't' } & \multirow{2}{*}{$\mathbf{P}$} & \multirow{2}{*}{ Results } \\
\hline & BT & AT & & & & & & \\
\hline Pain & 1.3 & 1.0 & 0.3 & 0.4030 & 0.1528 & 1.964 & 0.0811 & $\begin{array}{l}\text { Not } \\
\text { Significant }\end{array}$ \\
\hline
\end{tabular}

Table no. 5: Showing Statistical analysis of Pallor (Pandutava)

\begin{tabular}{|l|l|l|l|l|l|l|l|l|}
\hline \multirow{2}{*}{ Symptoms } & \multicolumn{2}{|c|}{ Mean } & \multirow{2}{*}{ Diff. } & SD & SE & ''t' & P & Results \\
\cline { 2 - 8 } & BT & AT & & & & \\
Pallor & 9.560 & 10.680 & -1.120 & 0.4733 & 0.1497 & 7.483 & 0.0001 & $\begin{array}{l}\text { Extremely } \\
\text { significant }\end{array}$ \\
\hline
\end{tabular}

Table no. 6: Showing Statistical analysis of burning sensation (Daha)

\begin{tabular}{|c|c|c|c|c|c|c|c|c|}
\hline \multirow{2}{*}{ Symptoms } & \multicolumn{2}{|c|}{ Mean } & \multirow{2}{*}{ Diff. } & \multirow{2}{*}{ SD } & \multirow{2}{*}{ SE } & \multirow{2}{*}{$' t^{\prime}$} & \multirow{2}{*}{$\mathbf{P}$} & \multirow[b]{2}{*}{ Results } \\
\hline & BT & AT & & & & & & \\
\hline $\begin{array}{l}\text { Burning } \\
\text { sensation }\end{array}$ & 0.4 & 0.0 & 0.4 & 0.5164 & 0.1633 & 2.449 & 0.0368 & Significant \\
\hline
\end{tabular}

Table no. 7: Showing Statistical analysis of weakness (Daurabalya)

\begin{tabular}{|l|l|l|l|l|l|l|l|l|}
\hline \multirow{2}{*}{ Symptoms } & \multicolumn{2}{|l|}{ Mean } & \multirow{2}{*}{ Diff. } & \multirow{2}{*}{ SD } & SE & ' $\mathbf{t}$ ' & P & Results \\
\cline { 2 - 9 } & BT & AT & 0.6 & 0.6992 & 0.2211 & 2.174 & 0.0239 & Significant \\
\hline Weakness & 0.6 & 0.0 & 0.6 &
\end{tabular}

EFFECT OF THE TRIAL DRUG OF GROUP -2 (SY) ON VARIOUS SUBJECTIVE SYMPTOMS

Table no. 8 Showing Statistical analysis of amount of bleeding (in no. of pads)

\begin{tabular}{|c|c|c|c|c|c|c|c|c|}
\hline \multirow{2}{*}{ Symptoms } & \multicolumn{2}{|c|}{ Mean } & \multirow{2}{*}{ Diff. } & \multirow{2}{*}{ SD } & \multirow{2}{*}{ SE } & \multirow{2}{*}{$' t^{\prime}$} & \multirow{2}{*}{$\mathbf{P}$} & \multirow{2}{*}{ Results } \\
\hline & BT & AT & & & & & & \\
\hline $\begin{array}{l}\text { Amount of } \\
\text { Bleeding }\end{array}$ & 3.4 & 1.6 & 1.8 & 1.989 & 0.6298 & 2.862 & 0.0187 & Significant \\
\hline
\end{tabular}


Table no. 9 Showing Statistical analysis of Duration of bleeding (in no. of days)

\begin{tabular}{|c|c|c|c|c|c|c|c|c|}
\hline \multirow{2}{*}{ Symptoms } & \multicolumn{2}{|c|}{ Mean } & \multirow{2}{*}{ Diff. } & \multirow{2}{*}{ SD } & \multirow{2}{*}{ SE } & \multirow{2}{*}{ 't' } & \multirow{2}{*}{$\mathbf{P}$} & \multirow{2}{*}{ Results } \\
\hline & BT & $\mathbf{A T}$ & & & & & & \\
\hline $\begin{array}{l}\text { Duration of } \\
\text { Bleeding }\end{array}$ & 5.7 & 3.1 & 2.6 & 3.373 & 1.067 & 2.438 & 0.0375 & Significant \\
\hline
\end{tabular}

Table no. 10 Showing Statistical analysis of intermenstrual bleeding (in no. of days)

\begin{tabular}{|c|c|c|c|c|c|c|c|c|}
\hline \multirow{2}{*}{ Symptoms } & \multicolumn{2}{|c|}{ Mean } & \multirow{2}{*}{ Diff. } & \multirow{2}{*}{ SD } & \multirow{2}{*}{ SE } & \multirow{2}{*}{$' t '$} & \multirow{2}{*}{$\mathbf{P}$} & \multirow{2}{*}{ Results } \\
\hline & BT & AT & & & & & & \\
\hline $\begin{array}{l}\text { Intermenstrual } \\
\text { Bleeding }\end{array}$ & 8.9 & 8.4 & 0.5 & 3.689 & 1.167 & 0.4286 & 0.6783 & $\begin{array}{l}\text { Not } \\
\text { Significant }\end{array}$ \\
\hline
\end{tabular}

Table no. 11 Showing Statistical analysis of Pain

\begin{tabular}{|l|l|l|l|l|l|l|l|l|}
\hline \multirow{2}{*}{ Symptoms } & Mean & Diff. & SD & SE & 't' & P & Results \\
\cline { 2 - 8 } & BT & AT & 0.9 & 0.4216 & 0.1333 & 1.5 & 0.1679 & $\begin{array}{l}\text { Not } \\
\text { Significant }\end{array}$ \\
\hline Pain & 1.1 & 0.9 & 0.2 & &
\end{tabular}

Table no. 12 Showing Statistical analysis of Pallor

\begin{tabular}{|l|l|l|l|l|l|l|l|l|}
\hline \multirow{2}{*}{ Symptoms } & \multicolumn{2}{|l|}{ Mean } & \multirow{2}{*}{ Diff. } & SD & SE & ' $\mathbf{~}$ ' & \multirow{2}{*}{ P } & Results \\
\cline { 2 - 8 } & BT & AT & & & & \\
\hline Pallor & 5.120 & 5.240 & -0.120 & 0.1398 & 0.0442 & 2.714 & 0.0239 & Significant \\
\hline
\end{tabular}

Table no. 13 Showing Statistical analysis of burning sensation

\begin{tabular}{|c|c|c|c|c|c|c|c|c|}
\hline \multirow{2}{*}{ Symptoms } & \multicolumn{2}{|c|}{ Mean } & \multirow{2}{*}{ Diff. } & \multirow{2}{*}{ SD } & \multirow{2}{*}{ SE } & \multirow{2}{*}{$' \mathbf{t}^{\prime}$} & \multirow{2}{*}{$\mathbf{P}$} & \multirow{2}{*}{ Results } \\
\hline & BT & $\mathbf{A T}$ & & & & & & \\
\hline $\begin{array}{l}\text { Burning } \\
\text { sensation }\end{array}$ & 0.4 & 0.0 & 0.4 & 0.5164 & 0.1633 & 2.4495 & 0.0368 & Significant \\
\hline
\end{tabular}

Table no. 14 Showing Statistical analysis of weakness

\begin{tabular}{|l|l|l|l|l|l|l|l|l|}
\hline \multirow{2}{*}{ Symptoms } & \multicolumn{2}{|l|}{ Mean } & Diff. & SD & SE & ' $\mathbf{t}^{\prime}$ & P & \multirow{2}{*}{ Results } \\
\cline { 2 - 9 } & BT & AT & & & & & \\
\hline Weakness & 0.4 & 0.0 & 0.4 & 0.5164 & 0.1633 & 2.449 & 0.0368 & Significant \\
\hline
\end{tabular}

Effect of The Trial Drug Of Group - 3 (PI) On Various Subjective Symptoms

Table no. 15 Showing Statistical analysis of amount of bleeding (in no. of pads)

\begin{tabular}{|c|c|c|c|c|c|c|c|c|}
\hline \multirow{2}{*}{ Symptoms } & \multicolumn{2}{|c|}{ Mean } & \multirow{2}{*}{ Diff. } & \multirow{2}{*}{ SD } & \multirow{2}{*}{ SE } & \multirow{2}{*}{$' t '$} & \multirow[b]{2}{*}{$\mathbf{P}$} & \multirow{2}{*}{ Results } \\
\hline & BT & $\mathbf{A T}$ & & & & & & \\
\hline $\begin{array}{l}\text { Amount of } \\
\text { Bleeding }\end{array}$ & 4.1 & 1.8 & 2.3 & 1.703 & 0.5385 & 4.271 & 0.0021 & $\begin{array}{l}\text { Very } \\
\text { Significant }\end{array}$ \\
\hline
\end{tabular}

Table no. 16 Showing Statistical analysis of Duration of bleeding (in no. of days)

\begin{tabular}{|c|c|c|c|c|c|c|c|c|}
\hline \multirow{2}{*}{ Symptoms } & \multicolumn{2}{|c|}{ Mean } & \multirow{2}{*}{ Diff. } & \multirow{2}{*}{ SD } & \multirow{2}{*}{ SE } & \multirow{2}{*}{$' t^{\prime}$} & \multirow{2}{*}{$\mathbf{P}$} & \multirow{2}{*}{ Results } \\
\hline & BT & $\mathbf{A T}$ & & & & & & \\
\hline $\begin{array}{l}\text { Duration of } \\
\text { Bleeding }\end{array}$ & 7.4 & 4.9 & 2.5 & 1.716 & 0.5426 & 4.607 & 0.0113 & Significant \\
\hline
\end{tabular}


Table no. 17 Showing Statistical analysis of intermenstrual bleeding (in no. of days)

\begin{tabular}{|c|c|c|c|c|c|c|c|c|}
\hline \multirow{2}{*}{ Symptoms } & \multicolumn{2}{|c|}{ Mean } & \multirow{2}{*}{ Diff. } & \multirow{2}{*}{ SD } & \multirow{2}{*}{ SE } & \multirow{2}{*}{$' \mathbf{t}^{\prime}$} & \multirow{2}{*}{$\mathbf{P}$} & \multirow{2}{*}{ Results } \\
\hline & BT & AT & & & & & & \\
\hline $\begin{array}{l}\text { Intermenstrual } \\
\text { Bleeding }\end{array}$ & 6.4 & 7.2 & -0.8 & 2.530 & 0.8000 & 1.000 & 0.3434 & $\begin{array}{l}\text { Not } \\
\text { Significant }\end{array}$ \\
\hline
\end{tabular}

Table no. 18 Showing Statistical analysis of Pain

\begin{tabular}{|c|c|c|c|c|c|c|c|c|}
\hline \multirow{2}{*}{ Symptoms } & \multicolumn{2}{|l|}{ Mean } & \multirow{2}{*}{ Diff. } & \multirow[b]{2}{*}{ SD } & \multirow{2}{*}{ SE } & \multirow{2}{*}{ 't' } & \multirow{2}{*}{$\mathbf{P}$} & \multirow{2}{*}{ Results } \\
\hline & BT & AT & & & & & & \\
\hline Pain & 0.5 & 0.5 & 0 & 0 & 0 & 0.000 & $\mathrm{P}>0.999$ & $\begin{array}{l}\text { Not } \\
\text { Significant }\end{array}$ \\
\hline
\end{tabular}

Table no. 19 Showing Statistical analysis of Pallor (Pandutava)

\begin{tabular}{|c|c|c|c|c|c|c|c|c|}
\hline \multirow{2}{*}{ Symptoms } & \multicolumn{2}{|l|}{ Mean } & \multirow{2}{*}{ Diff. } & \multirow{2}{*}{ SD } & \multirow{2}{*}{ SE } & \multirow{2}{*}{ 't' } & \multirow{2}{*}{$\mathbf{P}$} & \multirow{2}{*}{ Results } \\
\hline & BT & AT & & & & & & \\
\hline Pallor & 5.080 & 5.080 & 0 & 0 & 0 & 0.000 & $\mathrm{P}>0.999$ & $\begin{array}{l}\text { Not } \\
\text { Significant }\end{array}$ \\
\hline
\end{tabular}

Table no. 20 Showing Statistical analysis of burning sensation (Daha)

\begin{tabular}{|c|c|c|c|c|c|c|c|c|}
\hline \multirow{2}{*}{ Symptoms } & \multicolumn{2}{|c|}{ Mean } & \multirow{2}{*}{ Diff. } & \multirow{2}{*}{ SD } & \multirow{2}{*}{ SE } & \multirow{2}{*}{$' t '$} & \multirow{2}{*}{$\mathbf{P}$} & \multirow{2}{*}{ Results } \\
\hline & BT & AT & & & & & & \\
\hline $\begin{array}{l}\text { Burning } \\
\text { sensation }\end{array}$ & 0.5 & 0.1 & 0.4 & 0.5164 & 0.1633 & 2.449 & 0.0368 & Significant \\
\hline
\end{tabular}

Table no. 21 Showing Statistical analysis of weakness (Daurabalya)

\begin{tabular}{|c|c|c|c|c|c|c|c|c|}
\hline \multirow{2}{*}{ Symptoms } & \multicolumn{2}{|c|}{ Mean } & \multirow{2}{*}{ Diff. } & \multirow{2}{*}{ SD } & \multirow{2}{*}{ SE } & \multirow{2}{*}{$' \mathbf{t}^{\prime}$} & \multirow{2}{*}{$\mathbf{P}$} & \multirow{2}{*}{ Results } \\
\hline & BT & AT & & & & & & \\
\hline Weakness & 0.2 & 0.2 & 00 & 00 & 00 & 0.000 & $\mathrm{P}>0.999$ & $\begin{array}{l}\text { Not } \\
\text { Significant }\end{array}$ \\
\hline
\end{tabular}

Table no. 22 Total Effect of Therapy in 30 Patients

\begin{tabular}{|l|l|l|l|}
\hline \multirow{2}{*}{ Result } & \multicolumn{3}{|c|}{ No. of Patients } \\
\cline { 2 - 5 } & $\begin{array}{l}\text { Group } \\
\text { (SYT) }\end{array}$ & $\begin{array}{l}\text { Group 2 } \\
\text { (SY) }\end{array}$ & Group 3 (PL) \\
\hline $\begin{array}{l}\text { Cured } \\
\text { (More than 75\%) }\end{array}$ & 06 & 01 & 03 \\
\hline $\begin{array}{l}\text { Marked Improvement } \\
\text { to 75\% relief) }\end{array} \quad(50$ & 01 & 05 & 01 \\
\hline $\begin{array}{l}\text { Moderate Improvement (25 to } \\
50 \% \text { relief) }\end{array}$ & 02 & 04 & 00 \\
\hline $\begin{array}{l}\text { No Improvement } \\
\text { (<25\% relief) }\end{array}$ & 01 & 00 & 06 \\
\hline
\end{tabular}




\section{DISCUSSION}

* The assessment of the results was made by adopting the standard methods of scoring the signs and symptoms. All the observations regarding the changes in the subjective parameters like the amount of bleeding, duration of bleeding, intermenstrual period and associated symptoms like body pain, pallor and burning sensation and weakness were assessed clinically and statistically.

* Effect on Amount of bleeding: On statistical analysis the result was extremely significant in Group 1(SYT) ( $<<0.0001)$ while in Group 3 (PL) it was very Significant and in Group 2 (SY) it found to be Significant. Clinically also it gives the corresponding result, the amount of bleeding is found to be reduced in all group.

* Effect on Duration of bleeding: On statistical analysis the result was extremely significant in Group 1(SYT) while in group 2 (SY) and 3 (PL) it is Significant.

* Effect on Intermenstrual bleeding: On Statistical analysis the result was significant in group 1 while in group 2 (SY) and group 3 (PL) it is not significant.

* Effect on Pain: On Statistical analysis the result was not significant in any of the trial group.

* Effect on Pallor: On statistical analysis the result was extremely significant in group 1(SYT) while in group in group 2 (SY) it found to be Significant and 3 (PL) it was not Significant.

* Effect on Burning Sensation: On statistical analysis the result was significant in all groups.

* Effect on Weakness: On statistical analysis the result was significant in group 1(SYT) and group 2 (SY) while in group 3 (PL) it was found not be Significant.

* Overall effect of therapy:

* Out of three Groups studied, in Group 1(SYT) marked improvement was seen in the one Patient, moderate improvement in two patients and 6 patients were completely cured. In Group 2(SY) marked improvement was seen in five cases, no improvement was seen in one case, moderate improvement was seen in four cases and one patient is completely cured. In Group 3 (PL) marked improvement was seen in one patient and moderate improvement was not seen in any case. No improvements were seen in six cases while three patients were completely cured.

* On comparing the effectiveness of the three Groups it was found that the relief in symptoms was clinically and statistically found to be significant.

* Utmost positive findings are seen in Group 1(SYT) where quite a lot of symptoms are completely cured rather than group 2(SY) and 3(PL), whereas group 3 also shows good results in curing symptoms but all symptoms are not totally alleviated.

* In comparison with Group 3(PL); Group 2(SY) illustrates immense results in healing the symptoms. It shows that in comparison of the results of the groups; Group 1(SYT) depicts an enormous outcome of Shatavari Yashti yoga.

* The results clearly indicate that Shatavari Yashti Yoga is effective remedy to cure Raktapradara (Abnormal uterine bleeding).

\section{CONCLUSION}

* Shatavari Yashti Yoga is found effective in Raktapradara (Abnormal uterine bleeding). It effectively reduced amount of bleeding, duration of bleeding, reduces pallor, and balya (weakness is reduced). 
* Overall effect of therapy concludes that the first group of the patients who have received Shatavari Yashti Yoga with Tandulodaka has shown highly significant results in comparison with rest of other groups.

* Shatavari Yashti Yoga with Tandulodaka is Non Hormonal, Cost Effective, Harmless and boon for the Patients of Raktapradara (Abnormal uterine bleeding).

\section{REFERENCES}

1. Kathleen A. Oriel ans Sarina S Charger, Abnormal Uterine Bleeding, Journal of American Family Physician, 1999, Oct 1; 60(5): 1371-1380.

2. Laxmipati Shastri Yogaratnakar Varanasi Chaukhamba Prakashan reprint 2009,Pg no 397

3. Mahajan B. K Methods in Biostatics New Delhi Leipzig Press $4^{\text {th }}$ Ed. 1984, Pg no. 126 